\section{Nuevo nombre para el Pneumocystis del humano: Pneumocystis jiroveci}

La primera descripción de Pneumocystis la hizo Chagas en 1909, pero los consideró una forma morfológica de Trypanosoma cruzi. En 1912 se estableció que se trataba de una nueva especie, denominada Pneumocystis carinii. Hasta finales de los años 80 se consideró que era un protozoo, pero el análisis del ADN demostró en 1988 que es un hongo, y después que los Pneumocystis de diferentes mamíferos son muy diferentes entre sí. Estos descubrimientos han llevado a un cambio de nomenclatura que se explica en este artículo. Con ese cambio, el Pneumocystis del ser humano (antes P. carinii f. sp. hominis) pasa a llamarse $P$. jiroveci (Frenkel 1999) y P. carinii se reserva para lo que antes era P. carinii f. sp. carinii.

En 1976, Frenkel ya había señalado las diferencias fenotípicas entre el Pneumocystis del ser humano y otros Pneumocystis, proponiendo el nombre de $P$. jiroveci. Sin embargo, el nombre no se publicó de forma válida y no tuvo aceptación. Más tarde, el análisis del ADN estableció definitivamente que el Pneumocystis del ser humano es muy diferente de los de otros animales. La reacción en cadena de la polimerasa (RCP) con cebadores que amplifican el ADN de todas las especies conocidas de Pneumocystis reveló que de las muestras de Pneumocystis procedentes de humanos solo se obtiene ADN de $P$. jiroveci. Además, tampoco se ha encontrado ADN de P. jiroveci en muestras pulmonares de ningún otro mamífero. La secuenciación de genes clonados confirmó estos datos. La secuencia de los genes de Pneumocystis procedentes del ser humano ha sido en todos los casos muy diferente de la de sus ortólogos de Pneumocystis procedentes de otras especies. Además, los experimentos realizados con ratas, ratones, hurones y monos han demostrado que las especies de Pneumocystis tienen huéspedes específicos.

Con esta información empezaron a aparecer propuestas de cambio de nombre, pero inicialmente se optó por una nomenclatura trinomial provisional, por temor a crear una falsa especie con los datos todavía escasos sobre la secuencia del ADN. Este sistema trinomial denominaba los diferentes tipos de Pneumocystis como formas especiales de $P$. carinii. Así, $P$. jiroveci se llamaba $P$. carinii formae specialis hominis ( $P$. carinii f. sp. hominis). Después se siguieron obteniendo datos sobre la secuencia del ADN y en el año 2001 ya no quedaban dudas de que el microorganismo que causa la neumonía por $P$. carinii en el ser humano debía ser reconocido como una especie distinta. Por entonces, el nombre $P$. jiroveci ya había sido publicado de forma válida en 1999, pero la publicación válida de un nombre no implica necesariamente que se use. Durante los Talleres Internacionales sobre Protistas Oportunistas que tuvieron lugar el año 2001 en Cincinnati, Estados Unidos, se reunieron cerca de 50 investigadores de todo el mundo para determinar si se debía mantener la nomenclatura trinomial o asignar nuevos nombres de especie. La decisión unánime fue dar nuevos nombres de especie (binomiales) a los microorganismos que entonces se conocían como formas especiales de $P$. carinii y sentar las normas para la creación de los nuevos nombres de especie. Desde entonces es incorrecto, biológica y taxonómicamente, denominar P. carinii al Pneumocystis procedente del ser humano. $P$. carinii se refiere exclusivamente al microorganismo conocido antes como $P$. carinii f. sp. carinii, una de las dos especies de Pneumocystis que solo se encuentran en la rata. En el futuro, para referirse al Pneumocystis del ser humano solo se debería usar el nombre $P$. jiroveci.

La principal objeción a esta nueva designación fue el problema que originaría este cambio de nombre en la literatura médica, donde la enfermedad causada por $P$. jiroveci es muy conocida por el acrónimo PCP (Pneumocystis carinii pneumonia). No obstante, el problema tiene fácil solución si se considera que, tanto en inglés como en español, las letras PC del acrónimo designan $\underline{\text { Pneumocystis, en }}$

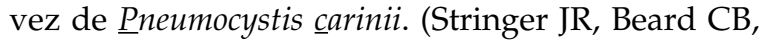
Miller RF, Wakefield AE. A new name (Pneumocystis jiroveci) for Pneumocystis from humans. Emerg Infect Dis 2002;8:891-896.)

\section{Enteritis por Escherichia coli O157:H7: tratamiento con antibióticos y riesgo de síndrome hemolítico urémico}

El síndrome hemolítico urémico (SHU) es un trastorno multisistémico caracterizado por anemia hemolítica, insuficiencia renal aguda y trombocitopenia. En su forma clásica suele aparecer tras una enteritis aguda, a menudo por cepas de Escherichia coli productoras de toxina Shiga. En Estados Unidos, la causa más frecuente de SHU es la infección 
entérica por E. coli, serotipo O157:H7. Esta complicación grave, que ocurre sobre todo en niños, tiene una mortalidad del 3 al 5\% y es la causa más frecuente de insuficiencia renal aguda en los niños de ese país. Entre sus posibles factores de riesgo, el más controvertido es el tratamiento de la enteritis aguda por E. coli O157:H7 con antibióticos, pues los estudios acerca de esta cuestión han incluido pequeñas muestras de pacientes, han utilizado diferentes regímenes de antibioticoterapia y han proporcionado resultados contradictorios. Este metaanálisis trata de determinar si la antibioticoterapia aumenta el riesgo de SHU en pacientes con enteritis por E. coli O157:H7.

Los estudios realizados en seres humanos se identificaron mediante una búsqueda bibliográfica en MEDLINE con las palabras clave hemolytic uremic syndrome, antibiotic, risk factor y Escherichia coli O157:H7. No se aplicaron restricciones de idioma. La búsqueda se limitó a los artículos publicados entre enero de 1983 (fecha de la descripción inicial de la asociación entre el SHU y las infecciones por E. coli productora de toxina Shiga) y febrero de 2002. Además se revisaron las listas de referencias de las publicaciones encontradas y se consultaron 12 expertos en el tema para que identificaran estudios adicionales no publicados. Los criterios de inclusión se definieron prospectivamente: análisis de una serie de pacientes con enteritis documentada por E. coli O157:H7, algunos con SHU; definición clara del SHU, y datos adecuados sobre la relación entre la antibioticoterapia y la aparición del SHU. Se calcularon las razones de los productos cruzados (odds ratios: OR) y sus intervalos de confianza del 95\% (IC95). La OR conjunta de los estudios analizados y su IC95 se calcularon con el modelo de efectos fijos de Mantel-Haenszel.

Se identificaron 26 estudios. Siete fueron excluidos por carecer de grupo de control, dos porque no definían claramente los criterios diagnósticos del $\mathrm{SHU}$, cuatro porque no investigaban sus factores de riesgo, tres porque carecían de datos sobre el uso de antibióticos como posible factor de riesgo, y uno porque incluía infecciones por otros serotipos de E. coli distintos del O157:H7. De los nueve estudios que cumplieron los criterios de inclusión, seis fueron retrospectivos, y tres prospectivos.

En cuatro de los seis estudios retrospectivos no se observó ninguna asociación y en otro se detectó un efecto protector de los antibióticos, aunque solo en el análisis univariado; solo uno mostró un efecto adverso, estadísticamente significativo, debido al uso de antibióticos. De los tres estudios prospectivos, uno mostró un aumento significativo del riesgo de SHU con el tratamiento antibiótico, otro un efecto protector de la fosfomicina, y el tercero ninguna asociación entre el uso de antibióticos y el SHU.
La OR conjunta de todos los estudios analizados fue de 1,15 (IC95: 0,79 a 1,68), lo cual indica que el uso de antibióticos no se asocia al SHU. La prueba de heterogeneidad (Breslow-Day) fue altamente significativa $(P<0,001)$. A ello contribuyó la heterogeneidad de las poblaciones estudiadas (adultos o niños), de los tipos de antibióticos administrados, de la duración de los tratamientos y de los métodos usados para tener en cuenta el efecto de la gravedad de la infección. La mayor parte de la heterogeneidad se debía a solo dos estudios. Una vez eliminados del análisis, la heterogeneidad dejó de ser significativa. No se identificaron sesgos de publicación considerables.

Este estudio no demostró que la administración de antibióticos en la enteritis por E. coli O157:H7 se asocie a un aumento del riesgo de SHU, aunque hay que tener en cuenta dos importantes limitaciones. La primera, que no se calculó una OR conjunta ajustada en función de la gravedad de la enfermedad porque tres de los nueve estudios no lo hicieron y los otros seis usaron métodos considerablemente diferentes. Esto hubiera sido importante, dado que los pacientes más graves tienen mayores probabilidades tanto de padecer SHU como de recibir antibióticos, introduciéndose así un posible factor de confusión en la relación entre el SHU y el uso de antibióticos. Por otra parte, los estudios in vitro y en animales indican que el momento de la instauración de la antibioticoterapia, su duración y el tipo de antibióticos usados pueden tener gran influencia en el riesgo de SHU, pero tampoco se pudieron analizar estos factores.

La decisión de administrar o no antibióticos es importante, sobre todo teniendo en cuenta sus grandes beneficios en la enteritis por Campylobacter jejuni, la diarrea del viajero por E. coli enterotoxigénica y la shigellosis. Como las manifestaciones de estas enfermedades no permiten distinguirlas de la enteritis por E. coli O157:H7, el aplazamiento de la antibioticoterapia hasta que se descarte la infección por E. coli O157:H7 puede tener efectos adversos en muchos pacientes. Para dilucidar definitivamente la relación entre el SHU y la antibioticoterapia de la enteritis por E. coli O157:H7 sería necesario un ensayo clínico aleatorizado con suficiente potencia estadística, en el que estuvieran representadas múltiples cepas diferentes de E. coli O157:H7. (Safdar N, Said A, Gangnon RE, Maki DG. Risk of hemolytic uremic syndrome after antibiotic treatment of Escherichia coli O157:H7 enteritis. A meta-analysis. JAMA 2002;288:996-1001.)

\section{La viudez como factor de riesgo de suicidio}

El suicidio es un problema grave de salud pública. En 1990 era una de las diez primeras causas 
de muerte en los países industrializados y en el año 2000 fue la undécima causa de muerte en los Estados Unidos. Los resultados de las investigaciones realizadas durante más de un siglo respaldan la hipótesis de que el matrimonio constituye un factor protector frente al suicidio. Por otra parte, el divorcio se ha asociado uniformemente a un aumento del riesgo, pero las asociaciones con la soltería y la viudez han sido menos uniformes. Aunque en los viudos y viudas se han registrado tasas de suicidio 8 a 50 veces mayores que en la población general (113 a 660 por 100000 ), se ha prestado poca atención a este grupo en alto riesgo.

En este estudio se determinaron las tasas de suicidio en diferentes grupos clasificados en función del estado civil, edad (grupos de 5 años entre los mayores de 20), sexo y raza (blancos y negros), prestando especial atención a los que habían enviudado recientemente. Los datos sobre la mortalidad por suicidio se obtuvieron a partir de los expedientes de causas de muerte del Centro Nacional de Estadísticas Sanitarias (National Center for Health Statistics Multiple-Cause-of-Death Files) de los años 1991 a 1996. Las estimaciones de la población total en cada uno de estos años se obtuvieron de la Oficina del Censo de los Estados Unidos (US Census Bureau). Debido al escaso número de suicidios entre las mujeres negras, este grupo fue excluido del análisis.

La tasa de suicidio estaba muy aumentada en los viudos y viudas jóvenes, y en particular en los viudos jóvenes, tanto blancos como negros, en comparación con las viudas jóvenes de raza blanca. Entre los jóvenes ( 20 a 34 años), la tasa fue 17 veces mayor en los hombres blancos viudos, y 9 veces mayor en los hombres negros viudos, en comparación con los casados. Dicho de otro modo, cada año se suicidará 1 de cada 400 varones viudos de 20 a 35 años, en comparación con 1 de cada 9000 hombres casados de la población general. La tasa también fue elevada en los viudos en comparación con los hombres con otro estado civil en todos los grupos de menos de 50 años, pero sobre todo en los más jóvenes. En los jóvenes, la viudez parece asociarse a un mayor riesgo de suicidio que el divorcio, pero al aumentar la edad se invierte esta tendencia y el divorcio se asocia a mayores tasas de suicidio que la viudez. El punto de inflexión ocurre mucho antes en las mujeres blancas (entre los 40 y los 49 años) que en los hombres, blancos o negros (entre los 55 y los 65 años). Las personas casadas fueron las que presentaron menores tasas de suicidio a lo largo de toda la vida, exceptuando los grupos más jóvenes.

Este estudio tiene la ventaja de ser el que mayor muestra ha utilizado entre todos los que han investigado las tasas de suicidio en función del estado civil. Dada la facilidad para identificar a este grupo, el alto riesgo de suicidio de los viudos jóve- nes representa una oportunidad única para la prevención. No obstante, desde el punto de vista de la asignación de recursos para la prevención, hay que tener en cuenta que, a pesar de las elevadas tasas de suicidio de los varones jóvenes viudos, el número absoluto de suicidios en este grupo es relativamente pequeño (cerca de 90 suicidios al año en viudos blancos de menos de 40 años, frente a 1350 suicidios al año en hombres blancos de 65 años o más). En estudios futuros se deberían investigar los factores de riesgo y los factores protectores frente al suicidio en personas que han enviudado recientemente. (Luoma JB, Pearson JL. Suicide and marital status in the United States, 1991-1996: is widowhood a risk factor? Am J Public Health 2002;92: 1518-1522.)

\section{Tratamientos tópicos de las verrugas cutáneas}

Las verrugas extragenitales son inocuas en personas inmunocompetentes y en general desaparecen espontáneamente en cuestión de meses o años. A menudo se aconseja no tratarlas, pero muchos pacientes solicitan tratamiento. Existen numerosos tratamientos tópicos, pero ninguno es particularmente eficaz y hay un gran desconocimiento sobre la eficacia absoluta y relativa de todos ellos. Este estudio presenta los resultados de una revisión sistemática de los ensayos clínicos aleatorizados controlados sobre cualquier tratamiento tópico de las verrugas cutáneas no complicadas.

Se hicieron búsquedas bibliográficas en MEDLINE (de 1966 a mayo de 2000), en EMBASE (de 1980 a agosto de 2000) y en el registro Cochrane de estudios controlados (marzo de 1999). Además se revisaron las listas de referencias de los estudios identificados y de artículos de revisión recientes, y se contactaron los laboratorios farmacéuticos y algunos expertos en el tema. Se incluyeron todos los estudios en los que los participantes fueran asignados aleatoriamente a diferentes intervenciones. La principal variable de eficacia analizada fue la completa resolución de las verrugas. Debido a la heterogeneidad de los estudios se usó un modelo de efectos aleatorios.

Se identificaron 50 estudios, de los cuales solo dos se consideraron de buena calidad. La heterogeneidad de los métodos y de las unidades de análisis (pacientes o verrugas) dificultó la agrupación de los datos.

En 17 estudios hubo grupos tratados con placebo. La tasa media de curación con el placebo fue de $30 \%$ tras un promedio de 10 semanas.

En 13 estudios se investigó el ácido salicílico (AS) tópico. Se usaron diferentes preparaciones, con AS del 15 a 60\%. La agrupación de los datos de 
seis ensayos clínicos controlados con placebo proporcionó una tasa de curación de $75 \%$ con el AS, y de $48 \%$ con el placebo (razón de los productos cruzados [odds ratio: OR]: 3,9; intervalo de confianza del 95\% [IC95]: 2,4 a 6,4).

En 16 estudios se investigó la crioterapia. En la mayoría de ellos se compararon diferentes regímenes de crioterapia, y no la crioterapia frente a otros tratamientos o al placebo. Los datos combinados de dos estudios pequeños que compararon la crioterapia con el placebo o la ausencia de tratamiento no mostraron diferencias significativas en cuanto a la tasa de curación (OR: 0,8; IC95: 0,2 a 4,2). En otros dos estudios con mayor número de participantes no se observaron diferencias entre la crioterapia y el AS (OR: 1,2; IC95: 0,7 a 1,8).

Dos pequeños estudios que compararon la inmunoterapia tópica con dinitroclorobenceno y el placebo mostraron una mayor eficacia del tratamiento activo (tasa de curación del $80 \%$, frente al $38 \%$ con el placebo).

En cinco estudios sobre la bleomicina intralesional las tasas de curación oscilaron entre el 16 y el $94 \%$. En dos, estas tasas fueron mayores con la bleomicina que con el placebo, en uno fueron mayores con el placebo y en otro no mostraron diferencias significativas.

El fluoruracilo y los interferones intralesionales tienen un interés más que nada histórico. La mayoría de los estudios son de las décadas de los 70 y 80 y tienen métodos y diseños muy heterogéneos. En términos generales, ninguno de los dos tratamientos presentó una eficacia notable.

En cuatro estudios sobre diferentes tipos de tratamiento fotodinámico el éxito fue variable. En uno de ellos, bien diseñado, la tasa de curación fue de $56 \%$ para las verrugas tratadas con ácido aminolevulínico, frente a $42 \%$ para las tratadas con placebo. No obstante, en este estudio todos los participantes usaron también AS.

No se identificaron estudios aleatorizados sobre la eficacia del laser de dióxido de carbono, la excisión quirúrgica, el curetaje o cauterización, el formaldehído ni el podofilino y la podofilotoxina.

La mayoría de los estudios revisados eran de mala calidad y la heterogeneidad de los diseños, métodos y variables de eficacia utilizados impidieron en la mayoría de los casos la agregación de los datos de diferentes estudios. Esta escasez de datos de calidad impide un uso racional de los tratamientos tópicos de las verrugas comunes. El AS parece ser eficaz y seguro, pero no se obtuvieron pruebas de que cualquiera de los demás tratamientos proporcione ventajas en términos de curación o de efectos adversos. Aunque muchos creen que la crioterapia puede tener éxito cuando fracasa el AS, no hay pruebas sólidas de que así sea. La bleomicina intralesional es un tratamiento de tercera línea bastante popular entre algunos dermatólogos, pero las pruebas sobre su eficacia son escasas. La inmunoterapia tópica con dinitroclorobenceno debería reservarse para centros especializados, debido a sus efectos adversos. En resumen, hay buenas pruebas de que el AS tópico tiene un efecto terapéutico y también hay algunos datos favorables a la eficacia del dinitroclorobenceno, pero no existen datos que respalden la eficacia de ninguno de los demás tratamientos, incluida la crioterapia. (Gibbs S, Harvey I, Sterling J, Stark R. Local treatments for cutaneous warts: systematic review. BMJ 2002;325:461-464.)

\section{Rifapentina una vez a la semana frente a rifampicina dos veces a la semana en pacientes negativos al VIH}

Los tratamientos breves con regímenes que contienen rifampicina son muy eficaces para curar la tuberculosis (TBC). Tras una fase inicial de administración diaria, se pueden administrar dos veces a la semana, lo cual simplifica mucho el tratamiento y facilita su observación directa. Una sola toma a la semana facilitaría todavía más el tratamiento y reduciría en un $30 \%$ el número de contactos necesarios entre el paciente y el suministrador del tratamiento bajo observación directa. Sin embargo, el régimen semanal no es suficientemente eficaz con la combinación de isoniazida y rifampicina. La rifapentina es un derivado de la rifamicina con excelente actividad frente a Mycobacterium tuberculosis, tanto in vitro como en animales, y tiene mayor semivida sérica que la rifampicina (10 a $15 \mathrm{~h}$, frente a 2 a $3 \mathrm{~h}$ ), por lo que podría ser eficaz en una sola toma semanal. En este ensayo clínico multicéntrico, aleatorizado y no enmascarado, se comparó el régimen de rifapentina e isoniazida una vez a la semana frente al de rifampicina e isoniazida dos veces a la semana durante la fase de continuación (últimos 4 meses) del tratamiento de la TBC pulmonar durante 6 meses.

Los participantes fueron pacientes de 18 años o más, seronegativos para el virus de la inmunodeficiencia humana (VIH), con TBC pulmonar confirmada por cultivo. Se excluyeron las embarazadas y las mujeres lactantes, así como los casos con resistencia a la isoniazida o a la rifampicina, intolerancia a los fármacos estudiados, TBC del sistema nervioso central u osteoarticular, silicosis, hemoglobinemia $<70 \mathrm{~g} / \mathrm{L}$, plaquetas $<50 \times 10^{9} / \mathrm{L}$, aspartatoaminotransferasa sérica $\geq 3$ veces el valor normal, bilirrubina sérica $\geq 2,5$ veces el valor normal o creatinina $\geq 2$ veces el valor normal.

Todos los pacientes recibieron isoniazida, rifampicina, pirazinamida y etambutol o estreptomicina durante los primeros 2 meses de tratamiento, 
tras los cuales fueron asignados aleatoriamente al tratamiento con 16 dosis de $600 \mathrm{mg}$ de rifapentina y $15 \mathrm{mg} / \mathrm{kg}$ de isoniazida (máximo de $900 \mathrm{mg}$ ) una vez a la semana (grupo I), o con 32 dosis de $10 \mathrm{mg} / \mathrm{kg}$ de rifampicina (máximo de $600 \mathrm{mg}$ ) y $15 \mathrm{mg} / \mathrm{kg}$ de isoniazida (máximo de $900 \mathrm{mg}$ ) dos veces a la semana (grupo II). Todas las dosis fueron administradas bajo observación directa.

Los pacientes fueron examinados cada 4 semanas durante el tratamiento y a los 3, 6, 9, 12, 18 y 24 meses de su finalización; en todas estas ocasiones se obtuvo una muestra de esputo. La principal medida de eficacia fue el fracaso/recidiva, que incluyó el fracaso, clínico o con cultivo positivo; la recidiva, clínica o con cultivo positivo, y el fracaso por incumplimiento del tratamiento. Los análisis se realizaron por intención de tratar.

Se incluyeron 1004 pacientes (502 por grupo), de los cuales 928 completaron el tratamiento y 803 la totalidad del estudio. Los que no completaron el tratamiento fueron 31 en el grupo I y 45 en el grupo II; no hubo diferencias entre los dos grupos con respecto a las causas que llevaron a los pacientes a no completar el tratamiento. La tasa cruda de fracasos/ recidivas fue del $9,2 \%(46 / 502)$ en el grupo I y del $5,6 \%(28 / 502)$ en el grupo II (riesgo relativo [RR]: 1,64, intervalo de confianza del 95\% [IC95]: 1,04 a 2,58; $P=0,04)$. Mediante un análisis de regresión multivariada de riesgos proporcionales se identificaron cinco características que estaban asociadas de forma independiente con el aumento del riesgo de fracaso/recidiva: el cultivo de esputo positivo a los 2 meses (razón de riesgo instantáneo [hazard ratio: HR]: 2,8, IC95: 1,7 a 4,6); la presencia de cavitación en la radiografía de tórax (HR: 3,0; IC95: 1,6 a 5,9); el bajo peso (HR: 3,0; IC95: 1,8 a 4,9); la afectación pulmonar bilateral (HR: 1,8; IC95: 1,0 a 3,1) y el hecho de ser blanco de origen no hispánico (HR: 1,8; IC95: 1,1 a 3,0). En un modelo de regresión de Cox en el que solo se incluyeron el grupo tera- péutico y el resultado, la HR de fracaso/recidiva entre los grupos terapéuticos fue de 1,6 (IC95: 1,0 a $2,6 ; P=0,04)$. Añadiendo a este modelo la positividad del cultivo a los 2 meses y la presencia de cavitación, la diferencia entre los dos grupos dejó de ser significativa (HR: 1,34; IC95: 0,83 a 2,18; $P=0,23$ ). Las tasas de fracaso/recidiva fueron especialmente altas en los pacientes con múltiples factores de riesgo, como aquellos con cultivo de esputo positivo a los 2 meses y cavitación ( $26,8 \%$ en el grupo I y $21,8 \%$ en el grupo II).

De los 1004 participantes, 56 (6\%) fallecieron. No hubo muertes atribuibles a complicaciones del tratamiento. No hubo diferencias entre los grupos con respecto a la frecuencia de acontecimientos adversos de grado 4, de acontecimientos de grado 4 atribuibles a los tratamientos, de hepatotoxicidad de grado 3 o 4 ni de trombocitopenia grave. La proporción de pacientes que abandonaron el tratamiento por acontecimientos adversos fue similar en los dos grupos.

Estos resultados indican que el régimen basado en la rifapentina una vez a la semana es seguro y eficaz en el tratamiento de la TBC pulmonar en pacientes seronegativos para el VIH sin cavitación pulmonar en la radiografía de tórax. Además, indican que los datos clínicos, radiográficos y microbiológicos son útiles para identificar a los pacientes que corren mayor riesgo de fracaso terapéutico o recidiva con cualquiera de los dos regímenes. Los dos factores con mayor efecto sobre el riesgo de recidiva fueron la magnitud y gravedad de la afectación pulmonar (enfermedad bilateral y cavitación) y la respuesta a la fase de tratamiento intensivo con cuatro fármacos (cultivo positivo a los 2 meses). (The TBC Trials Consortium. Rifapentin and isoniazid once a week versus rifampicin and isoniazid twice a week for treatment of drug-susceptible pulmonary TBC in HIV-negative patients: a randomised clinical trial. Lancet 2002;360:528-34.) 